\title{
A descriptive study of random forest algorithm for predicting COVID-19 patients outcome
}

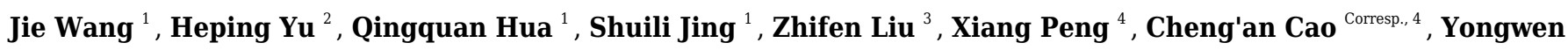 \\ Luo ${ }^{\text {Corresp. } 5}$ \\ ${ }^{1}$ Department of Otolaryngology-Head and Neck Surgery, Renmin Hospital of WUHAN University, Wuhan, Hubei, China \\ Department of Nail and breast surgery, Wuhan Forth Hospital, Wuhan, Hubei, China \\ 3 Department of Nephrology, Wuhan Forth Hospital, Wuhan, Hubei, China \\ 4 Department of Neurosurgery, Wuhan Forth Hospital, Wuhan, Hubei, China \\ 5 Department of Urology, Zhongnan Hospital of Wuhan University, Wuhan, Hubei, China \\ Corresponding Authors: Cheng'an Cao, Yongwen Luo \\ Email address: 2017202040101@whu.edu.cn, luoywen@whu.edu.cn
}

Background: The outbreak of coronavirus disease 2019 (COVID-19) that occurred in Wuhan, China, has become a global public health threat. It is necessary to identify indicators that can be used as optimal predictors for clinical outcomes of COVID-19 patients. Methods: The clinical information from 126 patients diagnosed with COVID-19 were collected from Wuhan Fourth Hospital. Specific clinical characteristics, laboratory findings, treatments, and clinical outcomes were analyzed from patients hospitalized for treatment from February 1 to March 15, 2020, and subsequently died or were discharged. A random forest (RF) algorithm was used to predict the prognoses of COVID-19 patients and identify the optimal diagnostic predictors for patients' clinical prognoses. Results: Seven of the 126 patients were excluded for losing endpoints, 103 of the remaining 119 patients were discharged (alive), and 16 died in the hospital. A synthetic minority over-sampling technique (SMOTE) was used to correct the imbalanced distribution of clinical patients. Recursive feature elimination (RFE) was used to select the optimal subset for analysis. Eleven clinical parameters, Myo, CD8, age, LDH, LMR, CD45, Th/Ts, dyspnea, NLR, D-Dimer, and CK were chosen with AUC approximately 0.9905. The RF algorithm was built to predict the prognoses of COVID-19 patients based on the best subset, and the area under the ROC curve (AUC) of the test data was $100 \%$. Moreover, two optimal clinical risk predictors, lactate dehydrogenase (LDH) and Myoglobin (Myo), were selected based on the Gini index. The univariable logistic analysis revealed a substantial increase in the risk for in-hospital mortality when Myo was higher than 80ng/ml (OR=7.54, $95 \% \mathrm{Cl}, 3.42$ to 16.63 ) and $\mathrm{LDH}$ was higher than 500U/L (OR=4.90, 95\% Cl, 2.13 to 11.25). Conclusion: We applied an RF algorithm to predict the mortality of COVID-19 patients with high accuracy and identified LDH higher than 500U/L and Myo higher than $80 \mathrm{ng} / \mathrm{ml}$ to be potential risk factors for the prognoses of COVID-19 patients in the early stage of the disease. 


\section{A descriptive study of random forest algorithm for}

\section{2 predicting COVID-19 patients outcome}

3 Jie Wang, MD ${ }^{1}$; Heping Yu, MD²; Qingquan Hua, MD ${ }^{1}$; Shuili Jing ${ }^{1}$; Zhifen Liu, MD ${ }^{3}$; Xiang

4 Peng, $\mathrm{MD}^{4}$; Cheng'an $\mathrm{Cao}^{*}, \mathrm{MD}^{4}$; Yongwen Luo *MD";

$5 \quad{ }^{1}$ Department of Otolaryngology-Head and Neck Surgery, Renmin Hospital of Wuhan

6 University, Wuhan, China

$7 \quad{ }^{2}$ Department of Nail and breast surgery, Wuhan Fourth Hospital, Wuhan, Hubei, China

$8 \quad{ }^{3}$ Department of Nephrology, Wuhan Fourth Hospital, Wuhan, Hubei, China

$9 \quad{ }^{4}$ Department of Neurosurgery, Wuhan Fourth Hospital, Wuhan, Hubei, China

$10{ }^{5}$ Department of Urology, Zhongnan Hospital of Wuhan University, Wuhan, 430073, China.

11 *Corresponding author:

12 Cheng'an Cao, Email: cca24@163.com.

13 Yongwen Luo, Email: luoywen@whu.edu.cn;

14 Address: No.473 Hanzheng Street, Qiaokou District, Wuhan, Hubei, China 


\section{Abstract}

Background: The outbreak of coronavirus disease 2019 (COVID-19) that occurred in Wuhan, China, has become a global public health threat. It is necessary to identify indicators that can be used as optimal predictors for clinical outcomes of COVID-19 patients.

Methods: The clinical information from 126 patients diagnosed with COVID-19 were collected from Wuhan Fourth Hospital. Specific clinical characteristics, laboratory findings, treatments, and clinical outcomes were analyzed from patients hospitalized for treatment from February 1 to March 15, 2020, and subsequently died or were discharged. A random forest (RF) algorithm was used to predict the prognoses of COVID-19 patients and identify the optimal diagnostic predictors for patients' clinical prognoses.

Results: Seven of the 126 patients were excluded for losing endpoints, 103 of the remaining 119 patients were discharged (alive), and 16 died in the hospital. A synthetic minority over-sampling technique (SMOTE) was used to correct the imbalanced distribution of clinical patients.

Recursive feature elimination (RFE) was used to select the optimal subset for analysis. Eleven clinical parameters, Myo, CD8, age, LDH, LMR, CD45, Th/Ts, dyspnea, NLR, D-Dimer, and CK were chosen with AUC approximately 0.9905 . The RF algorithm was used to predict the prognoses of COVID-19 patients based on the best subset, and the area under the ROC curve (AUC) of the test data was $100 \%$. Moreover, two optimal clinical risk predictors, lactate dehydrogenase (LDH) and Myoglobin (Myo), were selected based on the Gini index. The univariable logistic analysis revealed a substantial increase in the risk for in-hospital mortality when Myo was higher than $80 \mathrm{ng} / \mathrm{ml}(\mathrm{OR}=7.54,95 \% \mathrm{CI}, 3.42$ to 16.63$)$ and $\mathrm{LDH}$ was higher than $500 \mathrm{U} / \mathrm{L}(\mathrm{OR}=4.90,95 \% \mathrm{CI}, 2.13$ to 11.25$)$. 
40 Conclusion: We applied an RF algorithm to predict the mortality of COVID-19 patients with

41 high accuracy and identified LDH higher than 500U/L and Myo higher than 80ng/ml to be potential risk factors for the prognoses of COVID-19 patients in the early stage of the disease.

\section{Introduction}

In December 2019, an outbreak of acute respiratory syndrome coronavirus pneumonia occurred in Wuhan, Hubei Province, China (Phelan et al. 2020), and attracted intense attention worldwide.

The World Health Organization (WHO) named the virus, 2019-nCoV (COVID-19), based on its identification from a patient's pharyngeal swab sample (Lei et al. 2020; Organization 2020).

SAS-nCov 2 is a species of Coronavirus (CoV), which is a family of the largest, enveloped, single-stranded, positive-sense RNA viruses ( $\mathrm{Su}$ et al. 2016). The scientific community and infection control agencies face enormous challenges in controlling the increasing intensity of the COVID-19 pandemic. However, the disease has spread rapidly around the world. By June 26, 2020, COVID-19 had affected 213 countries, with over 9,621,470 confirmed cases and 487,295 deaths worldwide (COVID-19 Coronavirus - Update https://virusncov.comaccessed June 26, 2020). The least absolute shrinkage and selection operator (LASSO) regression has been used to identify the important factors of severity transition in COVID-19 patients (Liu et al. 2020), and critically ill patients exhibited respiratory failure, acute respiratory distress syndrome, heart failure, and septic shock, which increased the mortality of COVID-19 patients (Zhou et al. 59 2020a).

Previous studies showed that patients who were elderly and had diabetes, cardiovascular disease, 61 chronic respiratory diseases, or cancer presented an increased risk for COVID-19-related 62 mortality worldwide (Guan et al. 2020; Huang et al. 2020b; Ji et al. 2020; Wang et al. 2020; Wu 
\& McGoogan 2020; Zhou et al. 2020b). However, few models have been used to predict the mortality of COVID-19. Therefore, an effective and robust model is urgently required to predict the mortality of COVID-19 based on routine laboratory assessments and demographic information from COVID-19 patients. Timely detection of patients with high risk is of great significance and may contribute to optimizing the use of limited resources and delivering proper care.

Machine learning is widely used in medical diagnosis, and feature selection is an integral part of accurate data processing (Guyon \& Elisseeff 2003). RF is a type of machine learning that can analyze complex interactions between clinical characteristics and provide high classification accuracy using a set of decision trees (Touw et al. 2013). Therefore, we used a risk RF prediction model based on the outcomes of COVID-19 patients to predict the likelihood of recovery or continued deterioration and speculated on their prognoses, and corresponding disease control strategies need to be stressed to protect these patients against SAS-nCov 2.

\section{Patients and methods}

\section{Study design and participants}

This was a retrospective cohort analysis that included 126 patients, aged 27 to 87 years, from Wuhan Fourth Hospital. These patients were diagnosed with COVID-19 based on the World Health Organization's interim guidelines. Of the 126 patients, seven patients were excluded due to a lack of known clinical endpoints. The remaining 119 patients in this study were hospitalized for treatment from February 1 to March 15, 2020. This study was approved by the Ethics Committee of Wuhan Fourth Hospital (KY 2020-032-01). The Hospital Ethics Committee waived the informed consent from the study participants due to the high transmissibility of the 
86

disease.

Among the patients, the criteria used for discharge were as follows. The highest temperature returned to normal for more than three days. Chest CT imaging revealed significant inflammation absorption, and the respiratory symptoms had substantially improved. Two consecutive nucleic acid tests from throat swabs were negative, and the time interval between testing was at least one day. Finally, after evaluation and a unanimous decision by the expert team, a patient was discharged.

\section{Data collection}

Clinical information for all patients was obtained from electronic medical records in Wuhan Fourth Hospital by three independent researchers. Patient information, including exposure history, demographics, medical history, laboratory findings, co-morbidities, and clinical outcomes were collected and analyzed. If data were missing from the medical records, we obtained data from attending doctors or directly from the patients. Access was granted by the director of the hospital.

\section{Statistical analysis}

Descriptive data were compared using quartiles and medians, and the $\chi^{2}$ test or Fisher's exact test was used to analyze categorical data. The Kolmogorov-Smirnov test was used to analyze the normality of the data from discharged patients $(n=103)$. The Shapiro-Wilk test was employed to analyze the normality of the data from patients who died $(n=16)$. Subsequently, normally distributed laboratory results were analyzed using independent sample paired t-tests. The nonparametric Mann-Whitney-Wilcoxon test was used for data that did not exhibit a normal 
109 distribution. Univariable logistic analysis was used to analyze the risk of mortality caused by two

110 variables in survival and non-survival patients. All data were assessed using IBM SPSS, Version 11126.0 .

112

113 Variable selection and model construction

114 The flowchart for the research design is shown in Fig. S1. Based on the imbalanced distribution 115 of COVID-19 discharged versus deceased patients (106:13), a SMOTE procedure was employed

116 to adjust the data to achieve a final ratio of $1: 1(103: 103)$. Spearman correlation was used to

117 calculate the correlations among the essential variables, which were chosen based on statistical

118 analysis. RFE was used to screen out the discriminative subset of COVID-19 patient clinical

119 characteristics with 10 -fold cross-validation to avoid the redundant information. Then an RF

120 classification model was used to predict the mortality of COVID-19 patients with 5-fold cross-

121 validation and the mean value of the accuracy of mtry $=1$ was the highest at 0.846 , a bagging

122 algorithm was used to randomly collect the clinical characteristic for a total of 500 times, the

123 Gini index was the split criterion, and the nodesize of RF classification model was 1 . The clinical

124 data were divided into a training set and a test set with a ratio of approximately 4: $1(166: 40)$.

125 All data were processed using $\mathrm{R}$ studio ( $\mathrm{R}$ 3.6.3), the entire workflow was processed with the 126 caret package (http://CRAN.R- project.org/ package=caret) to keep the model construction and 127 validation consistent.

\section{Correlation analysis and assessment of accuracy}

130 A partial dependence correlation analysis was employed to provide a graphical depiction of the 131 marginal effect of a variable on the COVID-19 patients' outcome during the calculation process 
132 (Greenwell 2017). The function being plotted was defined as:

133

134

135

136

$$
\tilde{f}_{(x)}=\frac{1}{\mathrm{n}} \sum_{n=1}^{n} f\left(x, x_{i c}\right)
$$

where $\mathrm{x}$ is the variable corresponding to the chosen clinical characteristic, and $\mathrm{x}_{\mathrm{iC}}$ represents the other variables in the clinical information. The summand was the predicted logits $(\log$ of a fraction of votes) for classification:

$$
f(x)=\log _{\mathrm{Pk}}(\mathrm{x})-\frac{1}{k} \sum_{j=1}^{k} \log _{P j}(x)
$$

where $\mathrm{K}$ is the number of classes, and $\mathrm{P}_{\mathrm{j}}$ is the proportion of votes for class $\mathrm{j}$.

The accuracy of the test group to identify the final diagnostic capability of the RF classification algorithm was assessed using AUC, it also was applied to choose the optimal mtry and the best subset of clinical characteristics for the RF model performance.

\section{Results}

\section{Clinical demographics and outcomes of COVID-19 patients}

We described a cohort of 126 patients hospitalized at the Wuhan Fourth Hospital between Feb. 1st to Mar. 15th, 2020, of whom approximately half were classified as severely ill or critically ill. The patient clinical demographics and outcomes are shown in Table 1. We found that 48 patients (38.1\%) were older than 65, and the median patient age was 60 years (IQR 53-69.5), which showed the prevalence of COVID-19 in older adults. The incidence of COVID-19 infection was gender-neutral in that the proportions of male and female patients were nearly identical. COVID19 patients typically exhibited fever $(92.0 \%)$, and $39(34.8 \%)$ patients had peak temperatures above $39^{\circ} \mathrm{C}$ (Table 1). The most frequently observed symptoms of COVID-19 patients on admission were cough (75.4\%), followed by fatigue (58.7\%), dyspnea (55.6\%). In addition, many patients suffered from co-morbidities with hypertension $(34.9 \%)$ being the most common 
155

156

157

158

159

160

161

162

163

164

165

166

167

168

169

170

171

172

173

174

175

176

177

co-morbidity, followed by diabetes (16.7\%), cardiovascular and macrovascular disease (11.9\%).

During treatment, 83 patients $(65.9 \%)$ used nasal cannulas for supplemental oxygen, indicating

that a nasal cannula was useful for COVID-19 patients, two additional respiratory support

strategies that were used were noninvasive mechanical ventilation (NMV) (27.8\%), and invasive mechanical ventilation (IMV) (4.0\%). The criteria used to determine illness severity were based on the Novel Coronavirus Pneumonia Prevention and Control Program (6th edition) published by the National Health Commission of China (http://www.gov.cn/zhengce/zhengceku/202003/04/5486705/files/ae61004f930d47598711a0d4cbf874a9.pdf). Among the patients included in this study, in addition to seven patients who were excluded due to lack of known clinical endpoints, 103 (86.6\%) patients were cured, and $16(13.4 \%)$ patients died.

\section{Laboratory findings of COVID-19 patients at admission}

The initial laboratory findings included complete blood count, serum biochemical tests, coagulation profiles, and myocardial enzymes. All patients were assessed to determine whether they deviated significantly $(\mathrm{p}<0.05)$ from a normal range to evaluate the status of important organ functions. As seen in Table 2, more than $80 \%$ of the patients exhibited lymphopenia, especially for CD4+ and CD8+ T lymphocytes (91.3\%), which confirmed the previous study that SARS-CoV-2 infection damaged the immune system (Huang et al. 2020a). Approximately half of the patients exhibited decreased $\mathrm{Th} / \mathrm{Ts}$ ratios, which differed from Middle East respiratory syndrome(Park et al. 2017). C-reactive protein (CRP) was elevated in $85.6 \%$ of the patients, and procalcitonin (PCT) was slightly increased. COVID-19 infection also impaired coagulation functions in some patients. In this study, the prothrombin time (PT) was prolonged in approximately half of the patients, fibrinogen (FIB) was increased in two-thirds of the patients, 
and D-dimer was increased in $76.2 \%$ of the patients. The severely infected COVID-19 patients displayed a trend towards reduced platelet counts, a higher D-dimer level, and a higher rate of DIC occurrence. The myocardial enzymes showed that myocardial cell injury occurred in some patients, as $60 \%$ exhibited elevated B-type natriuretic peptide (BNP) and patients with elevated LDH accounted for $76.2 \%$ of the total.

\section{Comparison of clinical characteristics between discharged and deceased patients}

A comparison of clinical characteristics between discharged (alive) and deceased patients revealed the significant features that most likely caused deterioration in the deceased patients. As seen in Table 3, the patients in the deceased group were older than those in the discharged group $(\mathrm{p}<0.001)$, and the majority were males $(75 \%)$. The proportion of patients who experienced dyspnea was apparently increased from the onset of illness in the deceased group $(p=0.018)$. The deceased patients were more susceptible to respiratory failure, and the arterial blood gas parameters, $\mathrm{PCO} 2, \mathrm{PO} 2, \mathrm{SO} 2$, and oxygenation at admission, were significantly reduced in the deceased group. The laboratory analysis in this study revealed that more patients in deceased group exhibited elevated neutrophils and lymphopenia, which indicated a "divergence" between these two variables. Therefore, the neutrophil to lymphocyte ratio (NLR) was used to indicate the severity of illness in the patients. The NLR was remarkably elevated in the deceased group, while the lymphocyte to monocyte ratio (LMR) was decreased in the deceased group. The immune system damage was a risk factor for unfavorable outcomes of the disease. $\mathrm{T}$ lymphocytes significantly decreased in the deceased group, especially CD4+ and CD8+, as did the $\mathrm{Th} / \mathrm{Ts}$ ratio. Compared to discharged patients, the deceased patients underwent more frequent myocardial cell injury, as parameters reflecting heart function, including Myo, CK, and LDH, were significantly increased in the deceased group. Moreover, the inflammation-related indices, 
203

204

205

206

207

208

209

210

211

212

213

214

215

216

217

218

219

220

221

222

223

224

225

CRP $(p=0.080)$ and PCT $(p=0.009)$ were significantly higher in the deceased group.

\section{Correlation between clinical characteristics}

Because imbalanced data distribution affects the prediction accuracy of the RF model, and the ratio of discharged versus deceased patients was 103:16, a SMOTE algorithm was used to balance the data to select a more representative and informative subset of parameters for COVID-19 patients. STOME adjusted the ratio between these two groups to achieve a ratio of 1:1 (103:103). Moreover, redundancy of information also is likely to decrease the prediction performances of the RF classification model, so the correlations between variables should be taken into account in the process of feature selection (Paul et al. 2017). A Spearman correlation coefficient test was used to analyze the correlation between clinical characteristics of the COVID-19 patients (Spearman 2010). A heatmap was used to show the correlations between variables in the form of a matrix in Figure 1. Each element in the matrix was the correlation coefficient between the variables, and the range $[-1,1]$ was used to evaluate degree of correlation between two variables. When the correlation coefficient was greater than 0.8 , and the p-value was smaller than 0.05 , the correlation was determined to be strong(Paul et al. 2017), indicating that the factors were redundant variables. The analysis revealed that CD45 and CD4 had a high correlation of $0.84(\mathrm{p}<0.01)$, meanwhile, NLR and neutrophils also had a correlation of $0.84(\mathrm{p}<0.01)$. As for the redundancy of the clinical features, further processing was required to select the best subset for the RF model.

\section{Variable selection and RF classification model construction}

To select the optimal subset of clinical features, an RFE processed with 10-fold cross-validation 
226

227

228

229

230

231

232

233

234

235

236

237

238

239

240

241

242

243

244

245

246

247

248

was used to select the best subset. The RFE could eliminate the redundant and irrelative information from the COVID-19 patients and enhance the performance of the RF classification model (Darst et al. 2018). The results selected 11 clinical characteristics, Myo, CD8, age, LDH, LMR, CD45, Th/Ts, dyspnea, NLR, D-Dimer, and CK with the highest accuracy at 0.9905

(Figure 2A), which revealed the optimal complexity of the feature subset. Next, the RF classification model was used to predict the prognoses of COVID-19 patients based on the best subset. Five-fold cross-validation was used to identify the optimal mtry for the RF classification model, and the highest accuracy of classification was mtry=1 (Figure 2B), with the highest corresponding mean value of AUC at 0.846. Moreover, out-of-bag (OOB) error represented the generalization ability of the RF to calculate the proportion of misclassification. In Figure $\mathbf{2 C}$, the OOB error gradually decreased and stabilized as the forest size increased, and it finally reduced to less than 0.05 when the tree number reached 500. Meanwhile, death and survival errors gradually reduced to the same level as the OOB. The final diagnostic capability of the RF classification calculations was assessed using the test group's accuracy, which was $100 \%$ (Figure 2D), the threshold for the test data ROC was 0.385 .

\section{Identification of the important predictors for clinical outcomes}

As we know, the result of the RF classification model was obtained by selecting the results of the combined predictions among 500 decision trees, and the Gini index was the split criterion. RFGini is one of the best methods for feature ranking worldwide, especially for the top five predicted features (Menze et al. 2009). The larger the Gini coefficient became, the more important the information content of the independent variables. As shown in Figure $\mathbf{3 A}$, the variables that were ranked as important included Myo, age, LDH, CD8, CK, LMR, CD45, NLR, 
$249 \mathrm{Th} / \mathrm{Ts}$, D-dimer, and dyspnea. The top five variables were Myo, age, LDH, CD8, CK, among

250 them, we chose Myo and LDH as two laboratory parameters to assess risk and indicate the

251 prognoses for COVID-19 patients. The accuracy of the variables screened by the RF model is

252 shown in Figure 3B, and the accuracy of Myo ranked the highest and was followed by age and

253 NLR.

254

255

256

\section{Relationship between clinical characteristics and survival in COVID-19 patients}

To further analyze the role of LDH and Myo in affecting the survival of COVID-19 patients, we compared the mortality of patients who exhibited different levels of LDH and Myo. Using univariate logistic analysis, a substantial increase in the risk of in-hospital mortality with increased levels of Myo and LDH was observed (Figure 4A). Patients with increased Myo ( $\geq 80 \mathrm{ng} / \mathrm{ml}$ ) exhibited a 7.54 -fold (95\% CI: 3.42 to 16.63$)$ increase in mortality compared to patients with low Myo $(<80 \mathrm{ng} / \mathrm{ml})$. Similarly, patients with increased LDH $(\geq 500 \mathrm{U} / \mathrm{L})$ exhibited a 4.90-fold (95\% CI: 2.13 to 11.25$)$ increase in mortality compared to patients with low LDH (< 500U/L). The levels of LDH and Myo were compared in discharged and deceased groups

(Figure 4B). The median and IRQ for these two variables in the deceased group were higher than in the discharged group $(\mathrm{p}<0.001)$. The partial dependence plot showed the impact of Myo and LDH on survival when the marginal effects were controlled for in the RF classification. As Figure 4C shows, there was a significant negative correlation between survival and the levels of LDH or Myo. Specifically, increased levels of LDH and Myo were precursors to a poor prognosis for COVID-19 patients. To test the ability of LDH and Myo to predict the outcome of COVID-19 patients (Figure 4D), we observed that the AUC for Myo was 0.857 (95\%CI 0.74660.9694), and the threshold for the Myo ROC curve was $46.95 \mathrm{ng} / \mathrm{mL}$; the AUC for LDH was 
2720.807 (95\%CI 0.7149-0.9053), and the LDH ROC curve threshold was 327.5U/L. These clinical

273 features all had high accuracy for prognoses prediction of COVID-19 patients, but their accuracy

274 was lower than that produced by the RF classification model.

\section{Discussion}

277

278

279

280

281

282

283

284

285

286

287

288

289

290

291

292

293

294

The COVID-19 virus that occurred in Wuhan, China, is highly contagious, and a large number of exposed people have become critically ill. This study provided a comprehensive description of the demographics, comorbidities, and laboratory findings, of COVID-19 patients. Among the laboratory results, it was observed that lymphocytes, including CD4+ and CD8+, were decreased in $91.3 \%$ of patients, which confirmed that SARS-CoV-2 infection injured the human immune system. Thus, subsequent immune responses to this virus may exacerbate the disease response (Huang et al. 2020a). Approximately 40\% of the COVID-19 cases were severe, and the disease resulted in a $13.4 \%$ mortality rate. The mortality rate observed in this study was higher than the average rate observed in Wuhan, which was 4\% by March 24, 2020 (Du et al. 2020). This difference was likely due to the fact that only severe patients could be transferred to the hospitals designated to treat COVID-19 patients. During the patients' hospitalization for treatment, we found that early COVID-19 symptoms are insidious, but the disease progression is fast. Therefore, early prediction of COVID-19 patients' outcomes and adopting appropriate treatment are urgently required.

In this study, significant clinical features $(\mathrm{p}<0.05)$ were identified between discharged and deceased patients using statistical analysis. These clinical features were used for RF classification model, with SMOTE and a feature reduction technique RFE, to predict mortality of COVID-19 patients. The AUC of the RF model reached 100\%, demonstrating its robust 
295 prediction ability. Moreover, Myo and LDH were identified as two optimal predictors of

296 COVID-19 patients' outcomes with the Gini index.

297 Our current studies suggested that the deceased patients were susceptible to multiple organ

298 failure, especially heart and respiratory failure. There are several potential reasons for myocardial cell injury in COVID-19 patients, including systemic inflammatory responses, ACE2-targeted SAS-Cov-2 attacks on myocardial and lung cells, adverse effects of some antivirus drugs (Clerkin et al. 2020), and some underlying myocardial-damaging co-morbidities, such as diabetes and hypertension. Previous studies have reported that heart injury was common in patients with pneumonia (Marrie \& Shariatzadeh 2007). It was reported that elevated concentrations of Myo in venous blood could predict the severity of COVID-19 (McRae et al. 2020). Myo is a significant myocardial marker that is used in the clinical detection of patients with severe pneumonia. In this study, we found that $75 \%$ of the non-survivors, whose Myo concentration was higher than $80 \mathrm{ng} / \mathrm{mL}$, exhibited hypertension, which might have accelerated myocardial injury in patients with COVID-19. A partial correlation also indicated that as the Myo concentrations increased, survival decreased. Moreover, based on univariate logistic analysis, a high level of Myo above $80 \mathrm{ng} / \mathrm{mL}$ correlated with a high mortality rate $(61.5 \%)$ from COVID-19, and the risk of mortality was increased by 7.54 (95\% CI: 3.42 to 16.63 ), suggesting that increased concentrations of Myo potentially led to poor outcomes. ventilation, which leads to hypoxia and carbon dioxide retention(Yang et al. 2020b), which damages tissues(Yang et al. 2020a). On the other hand, microcirculation disorders caused by the 
318 infection and insufficient tissue perfusion also result in tissue damage. Both processes lead to

319 LDH accumulation in circulating blood. In our current research, the LDH values of $96(76.2 \%)$

320 patients were higher than the normal reference range, and the average level of LDH for non-

321 survivors was higher than survivors. LDH concentrations higher than 500U/L were associated

322 with high mortality risk $(\mathrm{OR}=4.90,95 \% \mathrm{CI}, 2.13$ to 11.25$)$. Moreover, in severely affected

323 patients, abnormal elevation of LDH often indicates rapid disease progression and acute

324 respiratory failure (Zhang et al. 2020). Therefore, an increase in LDH was a significant risk

325 factor for COVID-19 patient mortality.

326 Given the above research, our findings suggest that strategies to protect organ function should be emphasized to improve the patients' survival. Except for ensuring that they remain protected

328 from getting infected, doctors should evaluate the cardiac condition and the degree of complications of patients before and during treatment to avoid adverse side-effects from drugs used for COVID-19 therapies. Moreover, some ACE inhibitors (ACEi) or angiotensin receptor blockers (ARB), which are used to treat comorbidities in COVID-19 patients, might increase the level of ACE2 in myocardial cells, theoretically, leading to elevated risk for cardiac injury. Therefore, improving the method for patients' treatment should be considered in COVID-19 therapy.

This study has several limitations. First, due to the inclusion and exclusion of a large number of patients, it was inevitable that some important variables were omitted, such as smoking, a history of allergies, and others. Second, we only studied a few patients who exhibited relatively severe illness due to limited medical resources during the epidemic. Third, only patients with clear endpoints were included in the research, some patients who were still in hospital (alive) for treatment were not incorporated into this research, which may result in statistical biases. Last but 
341 not the least, about 5\% information was lost from the COVID-19 patient list, the missing count

342 variables were supplemented by the median, and the missing categorical variables were

343 supplemented by the mode, which led to biases of the clinical characteristics collection.

\section{Conclusion}

In this study, we described the clinical characteristics of COVID-19 patients during hospitalization and innovatively used an RF classification model with these clinical characteristics to predict COVID-19 prognoses. Moreover, we found that LDH concentrations that were higher than 500U/L, and Myo concentrations higher than $80 \mathrm{ng} / \mathrm{ml}$ could be identified as two potential risk factors for mortality of COVID-19 patients. Finally, appropriate treatment should be considered for patients with cardiac and tissue injury.

\section{Abbreviations}

RF: Random forest; ROC: receiver operating characteristic; AUC: Area under the ROC curve; IQR: Interquartile range; ARDS: Acute respiratory distress syndrome; Lym: Lymphocyte; Myo: Myoglobin; NMV: Noninvasive mechanical ventilation; IMV: Invasive mechanical ventilation; CRP: C-reactive protein; PCT: procalcitonin ; FIB: fibrinogen; BNP: B-type natriuretic peptide; CK-MB: creatine kinase-MB; AST: aminotransferase; ALT: alanine aminotransferase ;TG: triglyceride; LDH: Lactate dehydrogenase; LMR: Lymphocyte to monocyte ratio; NLR: Neutrophil to lymphocyte ratio; Mon: Monocyte; OOB: out-of-bag; Neu: Neutrophil.

\section{Acknowledgments}


We thank the patients and their family members for participating in our study. We appreciate all

the efforts of our colleagues in treating the patients.

\section{References}

Clerkin KJ, Fried JA, Raikhelkar J, Sayer G, Griffin JM, Masoumi A, Jain SS, Burkhoff D, Kumaraiah D, Rabbani L, Schwartz A, and Uriel N. 2020. COVID-19 and Cardiovascular Disease. Circulation. 141:1648-1655.

Darst BF, Malecki KC, and Engelman CD. 2018. Using recursive feature elimination in random forest to account for correlated variables in high dimensional data. BMC Genet. 19:65.

Du R-H, Liang L-R, Yang C-Q, Wang W, Cao T-Z, Li M, Guo G-Y, Du J, Zheng C-L, and Zhu QJERJ. 2020. Predictors of mortality for patients with COVID-19 pneumonia caused by SARSCoV-2: a prospective cohort study. Eur Respir J. 55 (5):2000524.

Germena G, Volmering S, Sohlbach C, and Zarbock A. 2015. Mutation in the CD45 inhibitory wedge modulates integrin activation and leukocyte recruitment during inflammation. J Immunol. 194:728-738.

Greenwell BMJRJ. 2017. pdp: An R Package for Constructing Partial Dependence Plots. $R$ Journal, 9(1): 421-436.

Guan W-j, Ni Z-y, Hu Y, Liang W-h, Ou C-q, He J-x, Liu L, Shan H, Lei C-l, and Hui DSJM. 2020. Clinical characteristics of 2019 novel coronavirus infection in China. $N$ Engl J Med. 382(18): 1708-1720.

Guyon I, and Elisseeff AJJomlr. 2003. An introduction to variable and feature selection. J Mach Learn Res. 3:1157-1182.

Huang C, Wang Y, Li X, Ren L, Zhao J, Hu Y, Zhang L, Fan G, Xu J, and Gu XJTI. 2020a. Clinical features of patients infected with 2019 novel coronavirus in Wuhan, China. Lancet. 395 (10223):497-506.

Huang R, Zhu L, Xue L, Liu L, Yan X, Wang J, Zhang B, Xu T, Ji F, and Zhao YJPNTD. 2020 . Clinical findings of patients with coronavirus disease 2019 in Jiangsu province, China: A retrospective, multi-center study. PLoS Negl Trop Dis. 14(5):e0008280.

Ingrisch M, Schöppe F, Paprottka K, Fabritius M, Strobl FF, De Toni EN, Ilhan H, Todica A, Michl M, and Paprottka PMJJoNM. 2018. Prediction of 90Y radioembolization outcome from pretherapeutic factors with random survival forests. $J$ Nucl Med. 59(5):769-773

Ji H-L, Zhao R, Matalon S, and Matthay MAJPr. 2020. Elevated plasmin (ogen) as a common risk factor for COVID-19 susceptibility. Physiol Rev. 100(3):1065-1075.

Lei Z, Cao H, Jie Y, Huang Z, Guo X, Chen J, Peng L, Cao H, Dai X, Liu JJTM, and Disease I. 2020. A cross-sectional comparison of epidemiological and clinical features of patients with coronavirus disease (COVID-19) in Wuhan and outside Wuhan, China. Travel Med Infect Dis. 35:101664.

Liu YP, Li GM, He J, et al. 2020. Combined use of the neutrophil-to-lymphocyte ratio and CRP to predict 7-day disease severity in 84 hospitalized patients with COVID-19 pneumonia: a retrospective cohort study. Ann Transl Med. 8(10):635.

Mamas MA, Fraser D, and Neyses LJIjoc. 2008. Cardiovascular manifestations associated with influenza virus infection. Int $J$ Cardiol. 130(3):304-309.

Marrie TJ, and Shariatzadeh MRJM. 2007. Community-acquired pneumonia requiring admission to an intensive care unit: a descriptive study. Medicine (Baltimore). 86(2):103-111.

McRae MP, Simmons GW, Christodoulides NJ, Lu Z, Kang SK, Fenyo D, Alcorn T, Dapkins IP, Sharif I, and Vurmaz DJLoaC. 2020. Clinical decision support tool and rapid point-of-care 
409

410

411

412

413

414

415

416

417

418

419

420

421

422

423

424

425

426

427

428

429

430

431

432

433

434

435

436

437

438

439

440

441

442

443

444

445

446

447

448

449

450

451

452

453

454

455

456

platform for determining disease severity in patients with COVID-19. Lab Chip. 20(12):20752085.

Menze BH, Kelm BM, Masuch R, Himmelreich U, Bachert P, Petrich W, and Hamprecht FA. 2009. A comparison of random forest and its Gini importance with standard chemometric methods for the feature selection and classification of spectral data. BMC Bioinformatics. 10:213.

Organization WH. 2020. Clinical management of severe acute respiratory infection when novel coronavirus ( $\mathrm{nCoV}$ ) infection is suspected: interim guidance, 25 January 2020. World Health Organization.

Park GE, Kang CI, Ko JH, Cho SY, Ha YE, Kim YJ, Peck KR, Song JH, and Chung DR. 2017. Differential Cell Count and CRP Level in Blood as Predictors for Middle East Respiratory Syndrome Coronavirus Infection in Acute Febrile Patients during Nosocomial Outbreak. $J$ Korean Med Sci. 32(1):151-154.

Paul D, Su R, Romain M, Sébastien V, Pierre V, and Isabelle G. 2017. Feature selection for outcome prediction in oesophageal cancer using genetic algorithm and random forest classifier. Comput Med Imaging Graph. 60:42-49.

Phelan AL, Katz R, and Gostin LO. 2020. The Novel Coronavirus Originating in Wuhan, China: Challenges for Global Health Governance. Jama. 323(8): 709-710.

Spearman C. 2010. The proof and measurement of association between two things. Int J Epidemiol. 39(5):1137-1150.

Su S, Wong G, Shi W, Liu J, Lai ACK, Zhou J, Liu W, Bi Y, and Gao GF. 2016. Epidemiology, Genetic Recombination, and Pathogenesis of Coronaviruses. Trends Microbiol. 24(6):490-502.

Touw WG, Bayjanov JR, Overmars L, Backus L, Boekhorst J, Wels M, and van Hijum SAJBib. 2013. Data mining in the Life Sciences with Random Forest: a walk in the park or lost in the jungle? Brief Bioinform. 14(3):315-326.

Wang D, Hu B, Hu C, Zhu F, Liu X, Zhang J, Wang B, Xiang H, Cheng Z, and Xiong YJJ. 2020. Clinical characteristics of 138 hospitalized patients with 2019 novel coronavirus-infected pneumonia in Wuhan, China. Jama. 323(11):1061-1069.

Warren-Gash C, Smeeth L, and Hayward ACJTLid. 2009. Influenza as a trigger for acute myocardial infarction or death from cardiovascular disease: a systematic review. Lancet Infect Dis. 9(10):601-610.

Wu Z, and McGoogan JMJJ. 2020. Characteristics of and important lessons from the coronavirus disease 2019 (COVID-19) outbreak in China: summary of a report of 72314 cases from the Chinese Center for Disease Control and Prevention. Jama. 323(13): 1239-1242.

Yang P, Wang P, Song Y, Zhang A, Yuan G, and Cui YJJoMV. 2020a. A retrospective study on the epidemiological characteristics and establishment of early warning system of severe COVID-19 patients. J Med Virol. 10.1002.

Yang X, Yu Y, Xu J, Shu H, Liu H, Wu Y, Zhang L, Yu Z, Fang M, and Yu TJTLRM. $2020 b$. Clinical course and outcomes of critically ill patients with SARS-CoV-2 pneumonia in Wuhan, China: a single-centered, retrospective, observational study. Lancet Respir Med. 8(5):475-481.

Zhang L, Yan X, Fan Q, Liu H, Liu X, Liu Z, and Zhang Z. 2020. D-dimer levels on admission to predict in-hospital mortality in patients with Covid-19. J Thromb Haemost. 18(6):1324-1329.

Zhou F, Yu T, Du R, Fan G, Liu Y, Liu Z, Xiang J, Wang Y, Song B, Gu X, Guan L, Wei Y, Li H, Wu X, Xu J, Tu S, Zhang Y, Chen H, and Cao B. 2020a. Clinical course and risk factors for mortality of adult inpatients with COVID-19 in Wuhan, China: a retrospective cohort study. Lancet. 395(10229):1054-1062.

\section{Figure legends}

Table 1. Demographic Characteristics and Clinical outcomes of Patients with COVID-19.

Peer] reviewing PDF | (2020:05:48501:2:0:NEW 9 Aug 2020) 
457 Table 2. Initial Laboratory Indices of Patients Infected With COVID-19.

458 Table3. Comparison of Clinical Characteristics between the Discharged and Deceased groups.

460 Figure 1. Spearman correlation analysis between clinical features of COVID-19 patents.

461 Heat map visualization shows Spearman correlation coefficients for clinical characteristics, the 462 variables have high correlations when $\rho>0.8$ and $p<0.05$. The analysis indicated that the 463 variables, CD45 and CD4, NLR and Neu, were highly correlated.

465 Figure 2. Variable selection and accuracy tests for the RF classification model.

466 (A) The process of choosing the best subset of clinical features. Eleven variables proved to be 467 the optimal selection with the corresponding highest accuracy (0.9905) of a predictive result 468 after 10-fold cross-validation.

(B) $M t r y=1$ was the best choice with the highest accuracy (0.846) for RF model prediction after 5-fold cross-validation.

(C) The OOB error rate to evaluate the quality of RF prediction of COVID-19 patients' outcomes. The middle line depicts the OOB error rate of all data. The top line shows the OOB error in the subgroup of survival patients and the bottom line shows OOB error in the COVID-19 patients. 
480 (A) Ranking of the clinical characteristics, according to Gini index.

481 (B) Ranking of the clinical characteristics based on the standardized drop in prediction accuracy. 482

Figure 4. Relationship between clinical characteristics and survival in COVID-19 patients.

(A) The table shows that the mortality increased significantly as the levels of Myo and LDH increased.

(B) The scatter plot shows the different concentrations of Myo and LDH in the non-survival and $487 \quad$ survival groups.

(C) The tendency chart shows the partial dependence correlation of Myo and LDH with survival. 


\section{Table $\mathbf{1}$ (on next page)}

Demographic Characteristics and Clinical outcomes of Patients with COVID-19.

Demographic Characteristics and Clinical outcomes of Patients with COVID-19. 
1 Table 1. Demographic Characteristics and Clinical Outcomes of patients with COVID-19.

\begin{tabular}{|c|c|}
\hline Variable & Number of patients (\%) \\
\hline No. of patients & 126 \\
\hline Age, median (IQR), y & $60(53-69.5)$ \\
\hline$\geq 65$ & $48(38.1)$ \\
\hline$<65$ & $78(61.9)$ \\
\hline Highest patient temperature, median (IQR), ${ }^{\circ} \mathrm{C}$ & $38.6(38-39)$ \\
\hline$\geq 39$ (high fever) & $39(34.8)$ \\
\hline$<39$ & $73(65.2)$ \\
\hline \multicolumn{2}{|l|}{ Gender } \\
\hline Male & $65(51.6)$ \\
\hline Female & $61(48.4)$ \\
\hline Contact history of epidiemic area & $7(5.6)$ \\
\hline \multicolumn{2}{|l|}{ Initial common symptoms } \\
\hline Cough & $95(75.4)$ \\
\hline Productive cough & $21(16.7)$ \\
\hline Hemoptysis & $6(4.8)$ \\
\hline Dyspnea & $70(55.6)$ \\
\hline Fatigue & $74(58.7)$ \\
\hline Myalgia & $41(32.5)$ \\
\hline Diarrhea & $14(11.1)$ \\
\hline \multicolumn{2}{|l|}{ Comorbidities } \\
\hline Hypertension & $44(34.9)$ \\
\hline Diabetes & 21(16.7) \\
\hline Cardriovascular and Macrovascular disease & $15(11.9)$ \\
\hline Liver and gall disease & $5(4.0)$ \\
\hline Nervous system disease & $6(4.8)$ \\
\hline Chronic lung disease & $13(10.3)$ \\
\hline Chronic kidney disease & $3(2.4)$ \\
\hline Endocrine system disease & $2(1.6)$ \\
\hline Immunological disease & $1(0.8)$ \\
\hline Hyperlipidemia & $3(2.4)$ \\
\hline Gastric disease & $7(5.6)$ \\
\hline Tumor & $6(4.8)$ \\
\hline \multicolumn{2}{|l|}{ Highest level of oxygen therapy } \\
\hline Nasal cannula & $83(65.9)$ \\
\hline NMV & $35(27.8)$ \\
\hline IMV & $5(4.0)$ \\
\hline IMV with ECMO & 0 \\
\hline \multicolumn{2}{|l|}{ Severity of clinical condnition } \\
\hline Moderate & $61(50.0)$ \\
\hline Severe & $38(31.1)$ \\
\hline Critical & $23(18.9)$ \\
\hline \multicolumn{2}{|l|}{ Clinical outcomes } \\
\hline Discharged alive & $103(86.6)$ \\
\hline Died & $16(13.4)$ \\
\hline
\end{tabular}

Abbreviations: IQR, interquartile range; NMV, noninvasive mechanical ventilation (including high flow supply and face mask); IMV, invasive mechanical ventilation; ECMO, extracorporeal membrane oxygenation. 


\section{Table 2 (on next page)}

Initial Laboratory Indices of Patients Infected With COVID-19.

Initial Laboratory Indices of Patients Infected With COVID-19. 
Table 2: Initial Laboratory Indices of Patients With COVID-19

2

\begin{tabular}{|c|c|c|c|c|}
\hline Laboratory Indices & $\begin{array}{l}\text { Reference } \\
\text { values }\end{array}$ & $\begin{array}{l}\text { Number of all } \\
\text { patients }\end{array}$ & Median (IQR) & $\begin{array}{l}\text { Number of patient with value } \\
\text { deviation from reference }(\%)\end{array}$ \\
\hline \multicolumn{5}{|l|}{ Hematology } \\
\hline White blood cells, $\times 109 / \mathrm{mL}$ & $3.5-9.5$ & 126 & $6.14(3.96-8.29)$ & $26(20.6)^{a}$ \\
\hline Neutrophils, $\times 109 / \mathrm{mL}$ & $1.8-6.3$ & 126 & $4.51(2.77-7.34)$ & $41(32.5)^{a}$ \\
\hline Lymphocytes, ×109/mL & $1.1-3.2$ & 126 & $0.73(0.53-1.01)$ & $102(81.0)^{b}$ \\
\hline Monocytes, $\times 109 / \mathrm{m} \mathrm{L}$ & $0.1-0.6$ & 126 & $0.29(0.20-0.43)$ & $6(4.8)^{a}$ \\
\hline NLR & NA & 126 & $5.99(3.07-12.59)$ & \\
\hline LMR & NA & 126 & $2.39(1.65-3.68)$ & \\
\hline CD4+ Tlym, $\times 106 / \mathrm{mL}$ & $450-1440$ & 116 & $142.16(78.50-271.84)$ & $108(93.1)^{b}$ \\
\hline CD8+ Tlym, $\times 106 / m L$ & $320-1250$ & 116 & $109.84(61.35-154.52)$ & $108(93.1)^{b}$ \\
\hline Th/Ts & $1.5-2.9$ & 116 & $1.52(0.96-2.08)$ & $55(51.9)^{b}$ \\
\hline $\mathrm{CD} 45, \times 106 / \mathrm{mL}$ & NA & 116 & 481.92(338.36-724.95) & \\
\hline \multicolumn{5}{|l|}{ Biochemical analysis } \\
\hline AST, U/L & $15-40$ & 126 & $27.5(19-44)$ & $48(38.1)^{a}$ \\
\hline ALT, U/L & $9-50$ & 126 & $25(15-43.5)$ & $22(17.5)^{b}$ \\
\hline $\mathrm{TG}, \mathrm{mmol} / \mathrm{L}$ & $0.45-1.69$ & 126 & $1.49(1.16-1.89)$ & $41(32.5)^{a}$ \\
\hline Creatine, $\mu \mathrm{M}$ & $57-111$ & 126 & $66(54-81.25)$ & $7(5.6)^{a}$ \\
\hline Tnl, $\mu \mathrm{g} / \mathrm{L}$ & $0-0.6$ & 91 & $0.03(0.03-0.03)$ & $1(1.1)^{a}$ \\
\hline Myo, ng/mL & $0-80$ & 111 & $27.2(18.1-38.05)$ & $13(11.7)^{a}$ \\
\hline $\mathrm{CK}, \mathrm{U} / \mathrm{L}$ & $0-171$ & 119 & $63.2(35.25-138.05)$ & $18(15.1)^{a}$ \\
\hline CK-MB, ng/m L & $0-2.37$ & 92 & $1.1(1-2.33)$ & $23(25.0)^{a}$ \\
\hline $\mathrm{BNP}, \mathrm{ng} / \mathrm{mL}$ & $0-100$ & 88 & $196.5(42.25-754.25)$ & $53(60.2)^{a}$ \\
\hline $\mathrm{CEA}, \mu \mathrm{g} / \mathrm{L}$ & $0-5$ & 57 & $2.08(1.51-5.53)$ & $15(26.3)^{a}$ \\
\hline $\mathrm{LDH}, \mathrm{U} / \mathrm{L}$ & $120-150$ & 126 & $306.50(241-389)$ & $123(97.6)^{a}$ \\
\hline \multicolumn{5}{|l|}{ Infection indices } \\
\hline $\mathrm{CRP}, \mathrm{mg} / \mathrm{L}$ & $0-5$ & 126 & $40.31(21.27-86.56)$ & $166(85.6)^{a}$ \\
\hline $\mathrm{PCT}, \mathrm{ng} / \mathrm{mL}$ & $0-0.5$ & 121 & $0.04(0.04-0.08)$ & $5(4.1)^{a}$ \\
\hline \multicolumn{5}{|l|}{ Coagulation function } \\
\hline $\mathrm{PT}, \mathrm{s}$ & $9-13$ & 126 & $13.6(11.3-41.2)$ & $61(48.4)^{a}$ \\
\hline APTT, s & $20-40$ & 126 & $35.5(22.4-69.9)$ & $22(17.4)^{a}$ \\
\hline $\mathrm{TT}, \mathrm{s}$ & $14-21$ & 126 & $16.3(12.8-72.3)$ & $3(2.4)^{a}$ \\
\hline INR & $0.8-1.25$ & 126 & $1.10(0.86-4.33)$ & $7(5.6)^{a}$ \\
\hline FIB, g/L & $2-4$ & 126 & $4.79(1.01-37.9)$ & $83(65.9)^{a}$ \\
\hline D-Dimer, mg/L & $0-0.2$ & 126 & $1.96(0.03-60.14)$ & $96(76.2)^{a}$ \\
\hline
\end{tabular}

aAbove reference; ${ }^{b}$ Below reference. 


\section{Table 3 (on next page)}

Comparison of Clinical Characteristics between the Discharged and Deceased groups.

Comparison of Clinical Characteristics between the Discharged and Deceased groups. 
1 Table3. Comparison of Clinical Characteristics between the Discharged and Deceased groups.

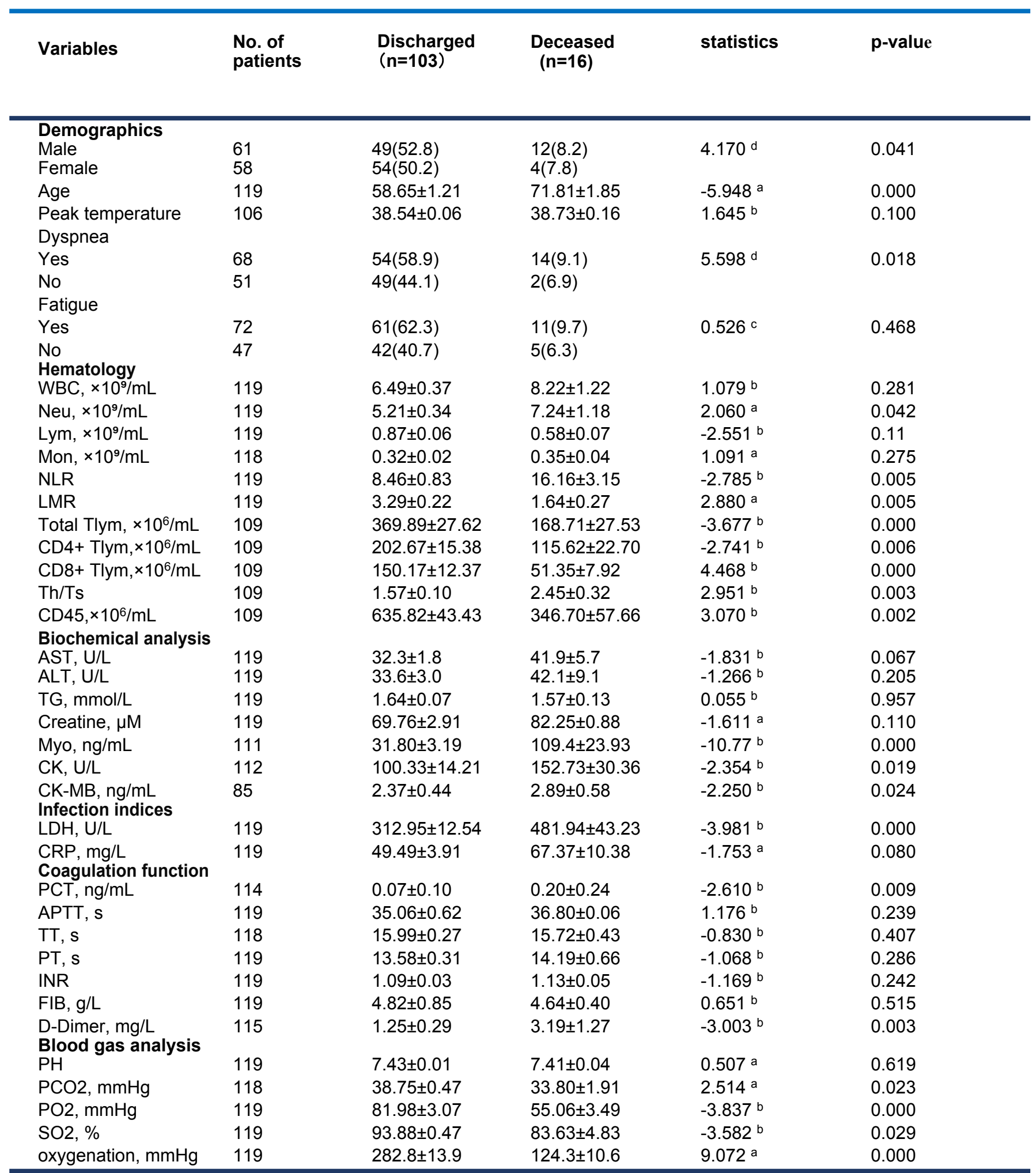

Abbreviations: NLR, neutrophil lymphocyte ratio; LMR, lymphocyte monocyte ratio;

a: t-test, b: Mann-Whitney $U$ test, c:X2 test, d:Continuity Correction 
Figure 1

Spearman correlation analysis between clinical features of COVID-19 patents.

Heat map visualization shows Spearman correlation coefficients for clinical characteristics, the variables have high correlations when $\rho>0.8$ and $p<0.05$. The analysis indicated that the variables, CD45 and CD4, NLR and Neu, were highly correlated.

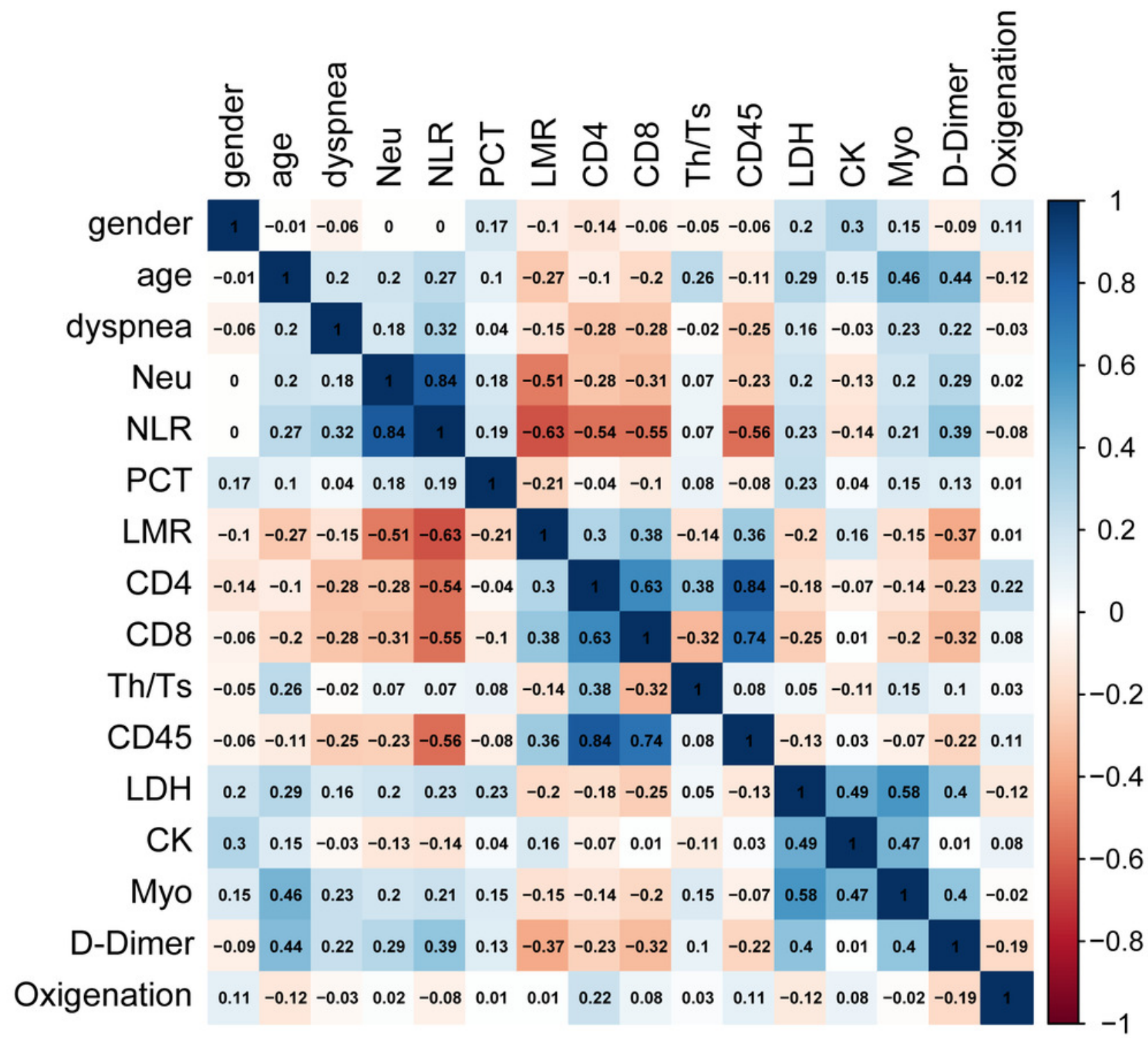




\section{Figure 2}

Variable selection and accuracy tests for the RF classification model.

(A) The process of choosing the best subset of clinical features. Eleven variables were proved to be the optimal selection with the corresponding highest accuracy (0.9905) of a predictive result after 10 -fold cross-validation. (B) Mtry=1 was the best choice with the highest accuracy (0.846) for RF model prediction after 5 -fold cross-validation. (C) The OOB error rate to evaluate the quality of RF prediction of COVID-19 patients' outcomes. The middle line depictes the OOB error rate of all data. The top line showes the OOB error in the subgroup of survival patients and the bottom line showes OOB error in the subgroup of dead patients. (D) ROC curve shows the accuracy of test data in the RF classification models, and the threshold of it is 0.385 

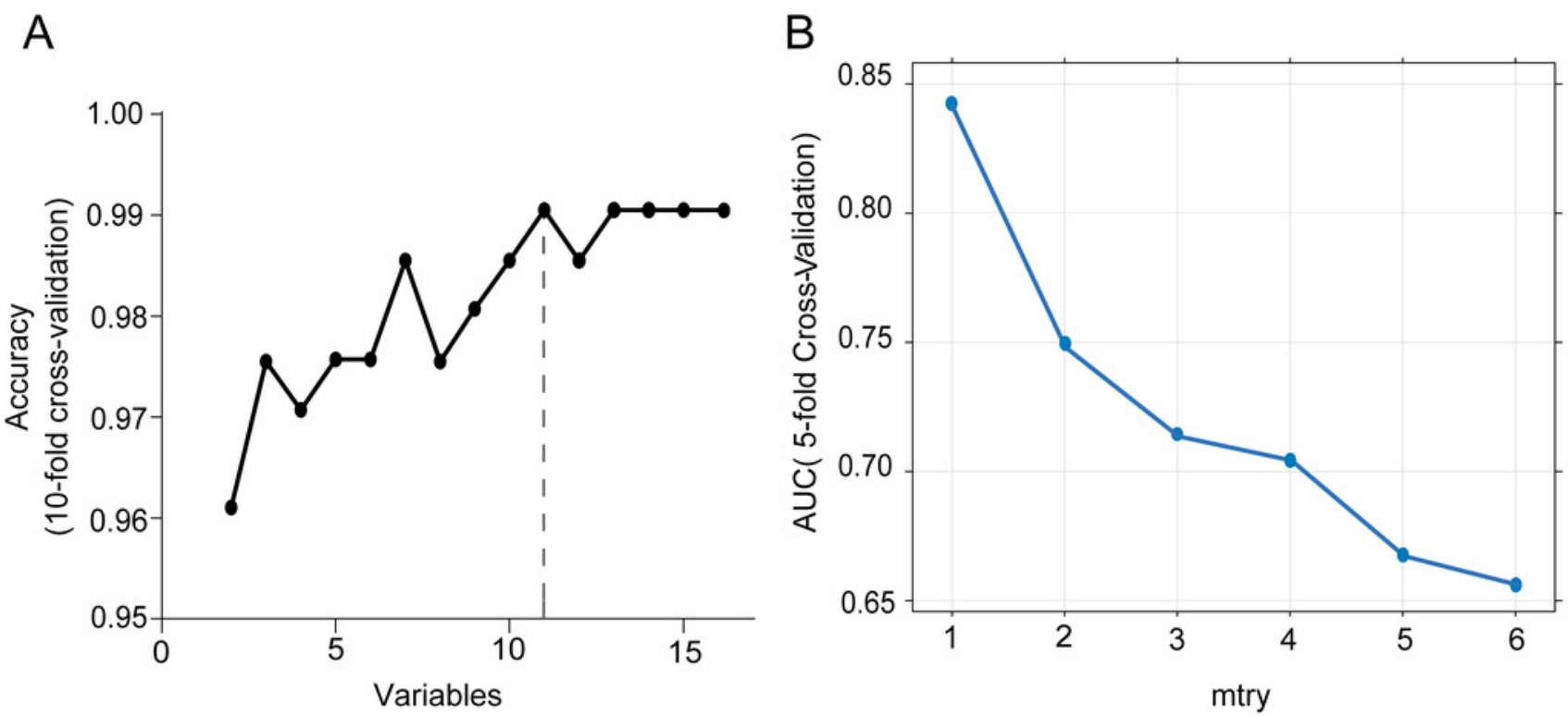

C

D
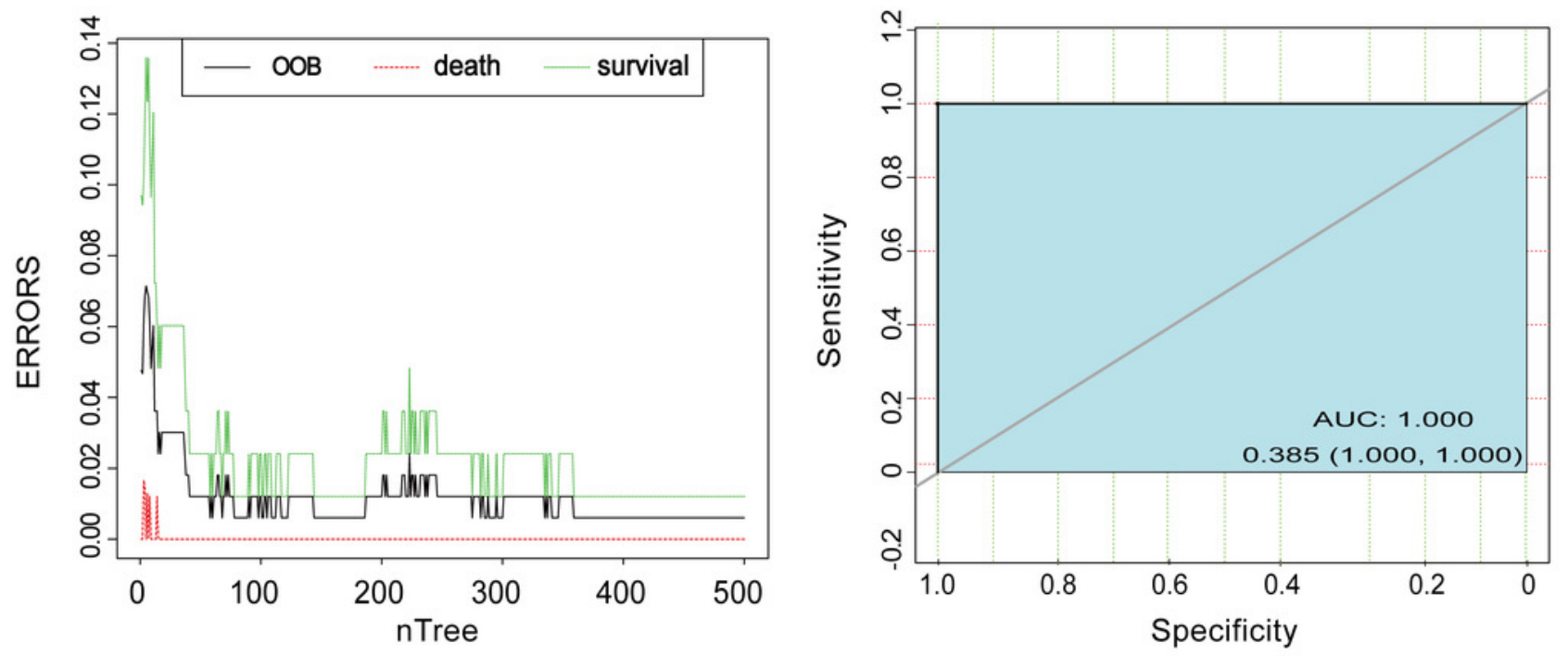
Figure 3

Identification of optimal diagnostic clinical characteristics for the prognoses of COVID-19 patients.

(A) Ranking of the clinical characteristics, according to Gini index. (B) Ranking of the clinical characteristics based on the standardized drop in prediction accuracy.
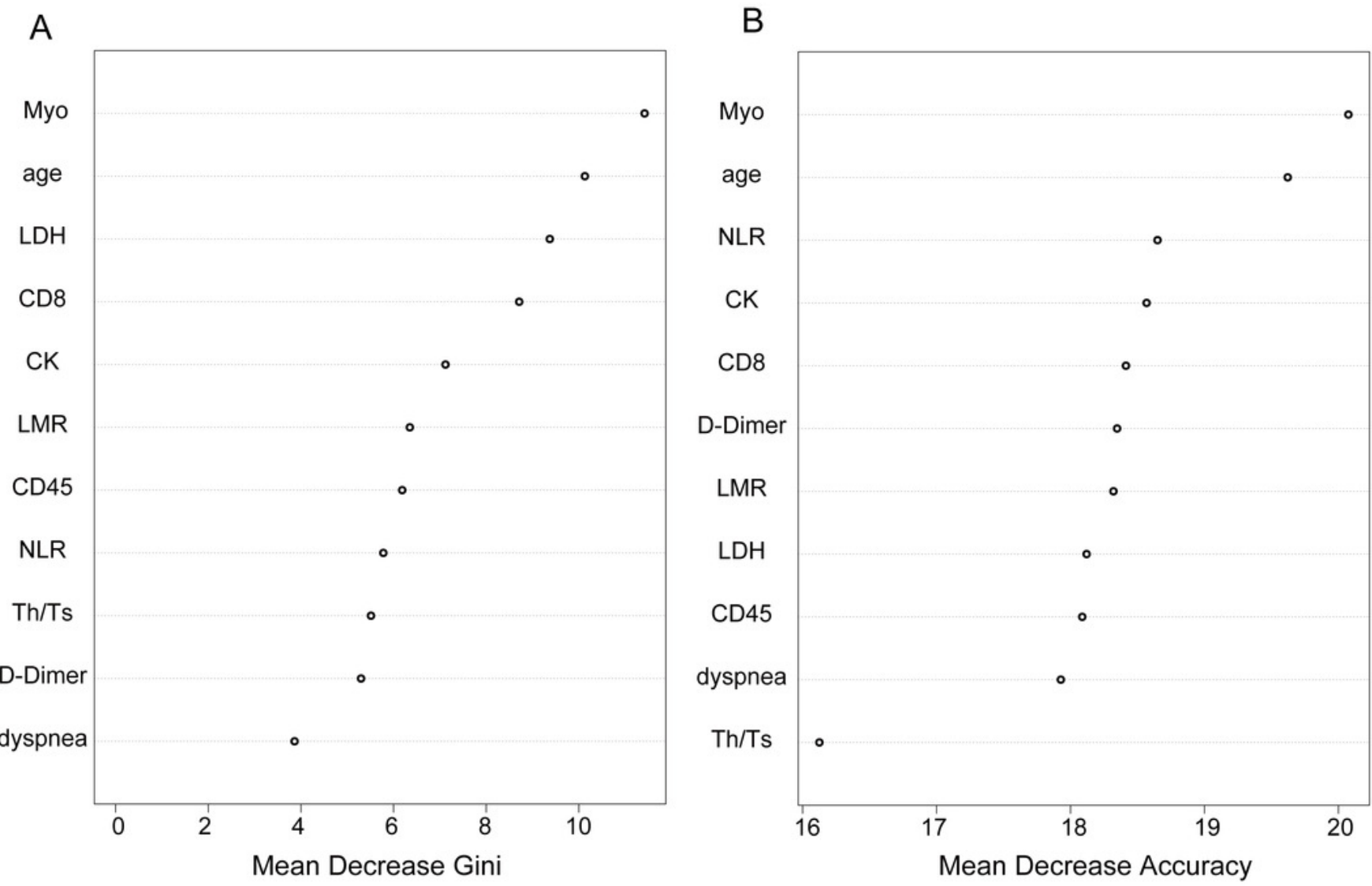
Figure 4

Relationship between clinical characteristics and survival in COVID-19 patients.

(A)The table shows that the mortality rate increased significantly as the levels of Myo and LDH increased. (B) The scatter plot shows the different concentrations of Myo and LDH in the non-survival and survival groups. (C)The tendency chart shows the partial dependence correlation of Myo and LDH with survival. (D) ROC curve shows the accuracy of Myo and LDH in predicting the COVID-19 patients' outcome.

A

\begin{tabular}{ccccccc}
\hline & Value & Cure & Death & Mortality & p-value & OR $(95 \% \mathrm{Cls})$ \\
\hline $\begin{array}{c}\text { Myoglobin } \\
(\mathrm{ng} / \mathrm{mL})\end{array}$ & $<80$ & 90 & 8 & $8.2 \%$ & & \\
LDH & $\geq 80$ & 5 & 8 & $61.5 \%$ & $<0.001$ & $7.54(3.42,16.63)$ \\
$(\mathrm{U} / \mathrm{L})$ & $<500$ & 96 & 10 & $9.4 \%$ & & \\
& $\geq 500$ & 7 & 6 & $46.2 \%$ & 0.013 & $4.90(2.13,11.25)$ \\
\hline
\end{tabular}

C

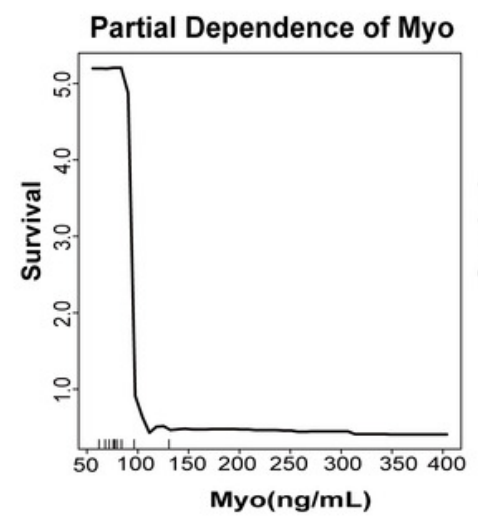

Partial Dependence of LDH

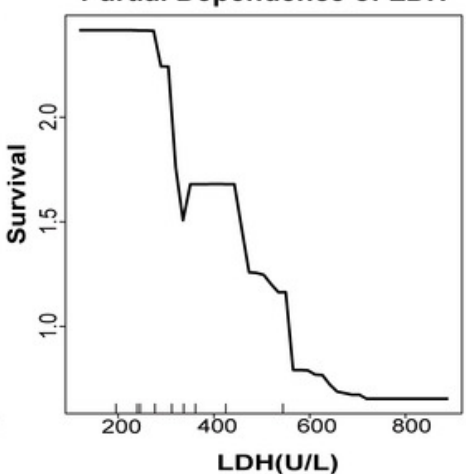

B
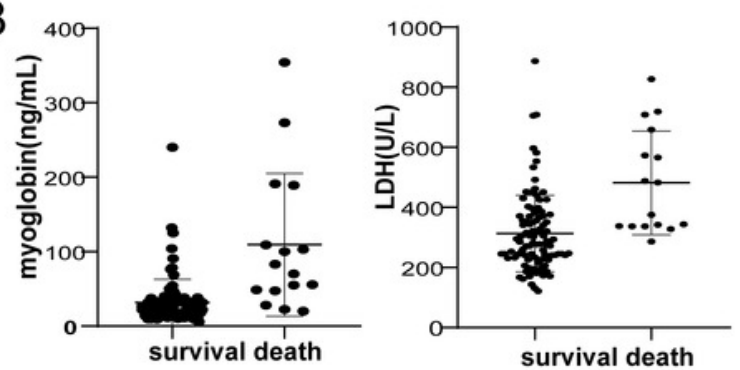

D
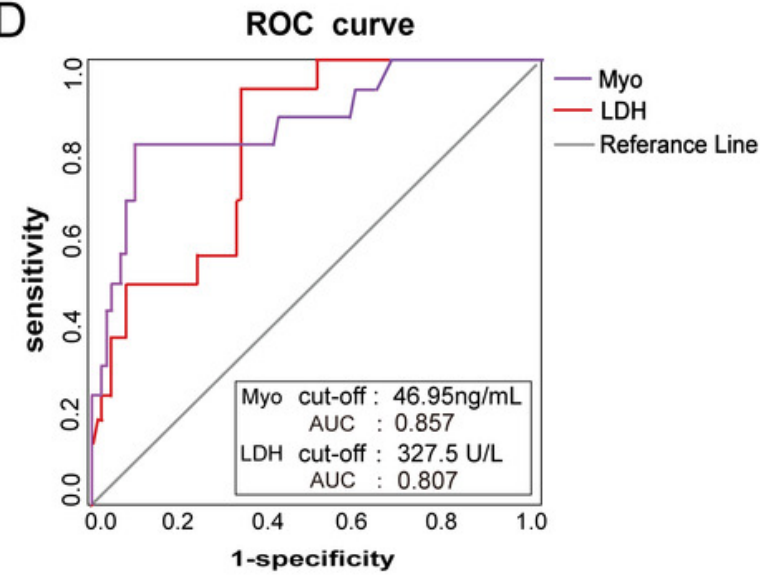